\title{
The Effects of Trapezoidal Groove on a Self-Acting Fluid-Lubricated Herringbone Grooves Journal Bearing
}

\author{
Jun Liu, ${ }^{1}$ and Yoshihiro Mochimaru ${ }^{2}$ \\ ${ }^{1}$ Tochigi R\&D Center, Keihin-Corp, Hoshakuji 2021-8, Takanezawa-Machi, Shioya-gun, Tochigi, Japan \\ ${ }^{2}$ Department of International Development Engineering, Graduate School of Science and Engineering, Tokyo Institute of Technology, \\ Meguro-ku, Tokyo 152, Japan
}

Correspondence should be addressed to Jun Liu; liujun3@hotmail.com

Received 12 May 2012; Accepted 10 July 2012

Academic Editors: J. Awrejcewicz, C.-H. Chien, M. Dular, J. H. Jang, F. Nair, and J. Wang

Copyright (C) 2013 J. Liu and M. Yoshihiro. This is an open access article distributed under the Creative Commons Attribution License, which permits unrestricted use, distribution, and reproduction in any medium, provided the original work is properly cited.

As a self-acting fluid-lubricated herringbone grooves journal bearing, a trapezoidal cross-sectional shape of grooves is considered. Trapezoidal groove shape effects on its bearing characteristics such as variations of load capacity, attitude, and friction torque for various trapezoidal angle of groove are determined.

\section{Introduction}

Since a herringbone grooves journal bearing has high stability at half whirl speed, it is equipped in miniature rotating machines. Performances of herringbone-grooved journal bearing are investigated by many researchers, for example, Vohr and Pan [1], Hamrock and Fleming [2], Murata et al. [3], Bonneau and Absi [4], Jang and Chang [5], as well as Liu and Yoshihiro [6].

In many cases numerical methods are applied to solve a pressure distribution equation (viz., a Reynolds equation of fluid thin film), evaluating fluid-lubricated herringbonegrooved journal bearing performance for load capacity and attitude angle.

In this paper, attention is focused on trapezoidal groove shape of a self-acting fluid-lubricated herringbone grooves journal-bearing to investigate influences of a variation of trapezoidal angle of groove change on characteristics.

\section{Analytical Model}

Consider a fluid-lubricated journal bearing equipped with herringbone grooves as shown in Figure 1. Let bearing length be $2 l$ and groove be symmetric with respect to its center of bearing. The shaft itself rotates around its center $O^{*}$ with an angular velocity $\omega$ in the counter-clockwise direction and revolves around the center $O$ of the bearing with an angular velocity $\Omega$ in the counter-clockwise direction. The eccentricity of the shaft is given by $\overline{O^{*}}=e$, and the outer bearing is fixed.

The inner radius of the bearing is $R_{b}$, the radius of the shaft corresponding to the plane without grooves is $R_{s 0}$, the bearing clearance $C_{r}$ is defined as $C_{r}=R_{b}-R_{s 0}$, and the groove depth, the groove width, ridge width, and grooves angle are denoted by $\delta, a_{g}, a_{r}$, and $\beta$, respectively. For a trapezoidal type of grooves, the trapezoidal angle $\psi$ is defined as shown in Figure 2.

Here, two coordinate systems are used, that is, $S(r, \theta, z)$, an inertial cylinder coordinate system, is fixed at the center $O$ of the outer bearing, and $S^{*}\left(r^{*}, \theta^{*}, z^{*}\right)$, noninertial cylinder coordinate system is fixed at the rotation shaft Relationship between the coordinates is given by

$$
\begin{gathered}
r \cos \theta=r^{*} \cos \left(\theta^{*}+\omega t\right)+e \cos \phi, \\
r \sin \theta=r^{*} \sin \left(\theta^{*}+\omega t\right)+e \sin \phi, \\
z=z^{*} .
\end{gathered}
$$

Hereafter, the superscript $*$ is meant for the noninertial coordinate system. The radial component $r$ or $r^{*}$ at the surface of the shaft is denoted by $R_{s}$ in $S$ or $R_{s}^{*}$ in $S^{*}$. 

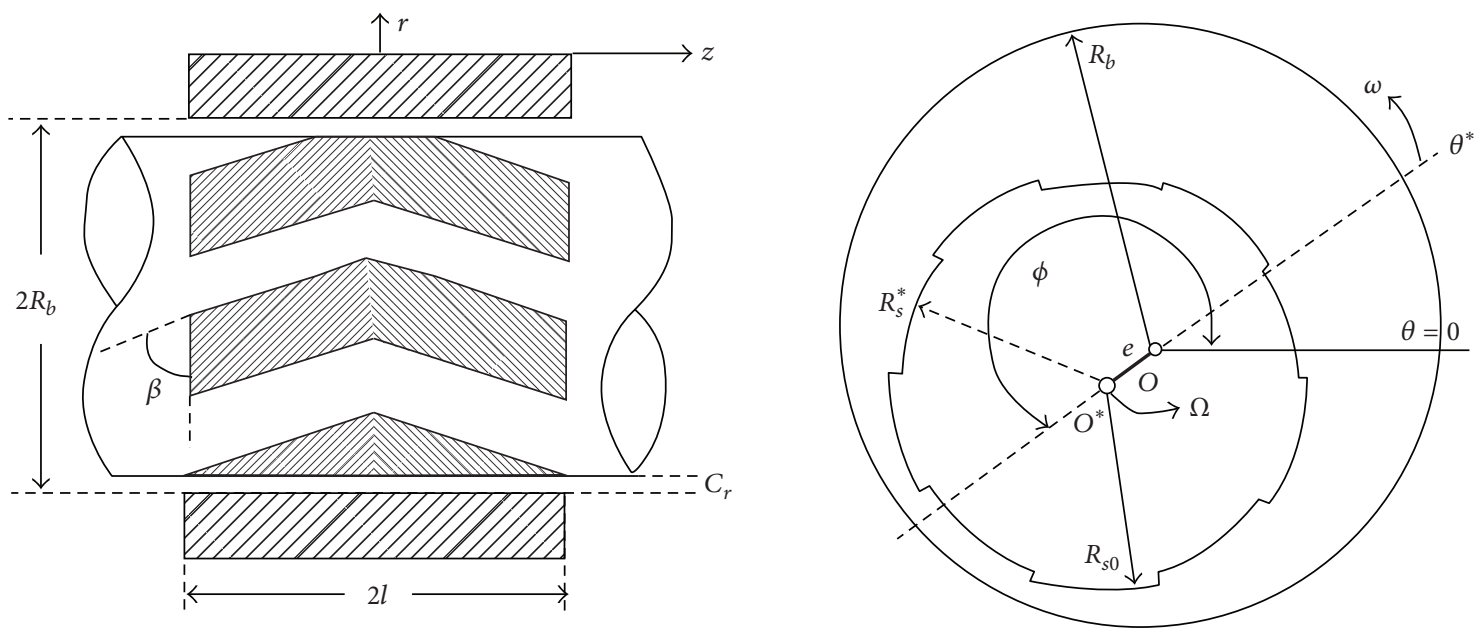

Figure 1: Herringbone-grooved journal bearing.

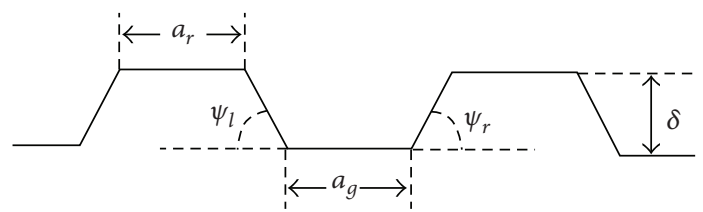

FIGURE 2: Cross-section perpendicular to the trapezoidal surface of groove.

\section{Local Velocity and Viscous Stresses}

The equation of motion of fluid in the bearing clearance is given under a lubrication approximation by

$$
\begin{gathered}
\frac{\partial^{2} v_{r}}{\partial r^{2}}+\frac{1}{r} \frac{\partial v_{r}}{\partial r}-\frac{v_{r}}{r^{2}}=0 \\
-\frac{1}{\eta r} \frac{\partial p}{\partial \theta}+\frac{\partial^{2} v_{\theta}}{\partial r^{2}}+\frac{1}{r} \frac{\partial v_{\theta}}{\partial r}-\frac{v_{\theta}}{r^{2}}=0 \\
-\frac{1}{\eta} \frac{\partial p}{\partial z}+\frac{\partial^{2} v_{z}}{\partial r^{2}}+\frac{1}{r} \frac{\partial v_{z}}{\partial r}=0 .
\end{gathered}
$$

Boundary conditions for velocity are

$$
\begin{array}{ccc}
v_{r}=u_{s}, & v_{\theta}=v_{s}, \quad v_{z}=0 \quad \text { at } r=R_{s}, \\
v_{r}=0, & v_{\theta}=0, \quad v_{z}=0 \quad \text { at } r=R_{b} .
\end{array}
$$

Integrating (2) under the boundary conditions (3) gives the local velocity distribution as

$$
v_{r}=-\frac{u_{s} R_{s}}{R_{b}^{2}-R_{s}^{2}}\left(r-\frac{R_{b}^{2}}{r}\right),
$$

$$
\begin{gathered}
v_{\theta}=\frac{1}{\eta} \frac{\partial p}{\partial \theta}\left[\frac{1}{2} r \ln \left(\frac{r}{R_{b}}\right)-\frac{1}{2}\left(r-\frac{R_{b}^{2}}{r}\right)\right. \\
\left.\times \frac{R_{s}^{2}}{\left(R_{b}^{2}-R_{s}^{2}\right)} \ln \left(\frac{R_{s}}{R_{b}}\right)\right] \\
-\frac{v_{s} R_{s}}{\left(R_{b}^{2}-R_{s}^{2}\right)}\left(r-\frac{R_{b}^{2}}{r}\right), \\
v_{z}=\left[\frac{r^{2}-R_{s}^{2}}{4}-\frac{R_{b}^{2}-R_{s}^{2}}{4} \frac{\ln \left(R_{s} / r\right)}{\ln \left(R_{s} / R_{b}\right)}\right] \frac{1}{\eta} \frac{\partial p}{\partial z} .
\end{gathered}
$$

The physical components of viscous stress in the lubrication fluid are given by

$$
\begin{gathered}
\tau^{r r} \equiv 2 \eta \frac{\partial v_{r}}{\partial r}, \\
\tau^{\theta \theta} \equiv 2 \eta\left(\frac{1}{r} \frac{\partial v_{\theta}}{\partial \theta}+\frac{v_{r}}{r}\right), \\
\tau^{r \theta} \equiv \eta\left[r \frac{\partial}{\partial r}\left(\frac{v_{\theta}}{r}\right)+\frac{1}{r} \frac{\partial v_{r}}{\partial \theta}\right], \\
\tau^{\theta z} \equiv \eta\left(\frac{1}{r} \frac{\partial v_{z}}{\partial \theta}+\frac{\partial v_{\theta}}{\partial z}\right), \\
\tau^{r z} \equiv \eta\left(\frac{\partial v_{r}}{\partial z}+\frac{\partial v_{z}}{\partial r}\right), \\
\tau^{\mathrm{zz}} \equiv 2 \eta \frac{\partial v_{z}}{\partial z}
\end{gathered}
$$


Substituting (4) into (5)-(7) at $r=R_{s}$ gives

$$
\begin{aligned}
& T_{R=R_{s / b}}^{R R}=-\frac{U_{s}}{R_{s / b}}\left(\frac{1+R_{s / b}^{2}}{1-R_{s / b}^{2}}\right) \\
& T_{r=R_{s}}^{\Theta \Theta}=-\left[\frac{1}{2 R_{s / b}}+\frac{\ln R_{s / b}}{R_{s / b}\left(1-R_{s / b}^{2}\right)}\right] \frac{2}{\sigma} \frac{\partial P}{\partial \Theta} \frac{\partial R_{s / b}}{\partial \Theta}+\frac{U_{s}}{R_{s / b}} \\
& +\frac{1}{R_{s / b}} \frac{\partial V_{s}}{\partial \Theta}+\frac{V_{s}}{R_{s / b}^{2}}\left(\frac{1+R_{s / b}^{2}}{1-R_{s / b}^{2}}\right) \frac{\partial R_{s / b}}{\partial \Theta}, \\
& T_{R=R_{\frac{s}{b}}^{R \Theta}}=\left(\frac{1}{2}+\frac{\ln R_{s / b}}{1-R_{s / b}^{2}}\right) \frac{1}{\sigma} \frac{\partial P}{\partial \Theta}+\frac{U_{s}}{2 R_{s / b}^{2}}\left(\frac{1+R_{s / b}^{2}}{1-R_{s / b}^{2}}\right) \frac{\partial R_{s / b}}{\partial \Theta} \\
& +\frac{1}{2 R_{s / b}} \frac{\partial U_{s}}{\partial \Theta}-\frac{V_{s}}{R_{s / b}}\left(\frac{1}{1-R_{s / b}^{2}}\right), \\
& T_{R=R_{s / b}}^{Z \Theta}=-\left(\frac{1}{2}+\frac{\ln R_{s / b}}{1-R_{s / b}^{2}}\right) \frac{1}{\sigma} \frac{\partial P}{\partial \Theta} \frac{\partial R_{s / b}}{\partial Z}+\frac{1}{2} \frac{\partial V_{s}}{\partial Z} \\
& +\frac{V_{s}\left(1+R_{s / b}^{2}\right)}{2 R_{s / b}\left(1-R_{s / b}^{2}\right)} \frac{\partial R_{s / b}}{\partial Z}-\frac{1}{2 \sigma} \frac{\partial P}{\partial Z} \frac{\partial R_{s / b}}{\partial \Theta} \\
& \times\left(1+\frac{1-R_{s / b}^{2}}{2 R_{s / b}^{2} \ln R_{s / b}}\right), \\
& T_{R=R_{s / b}}^{R Z}=\frac{1}{2} \frac{\partial U_{s}}{\partial Z}+\frac{U_{s}}{2 R_{s / b}}\left(\frac{1+R_{s / b}^{2}}{1-R_{s / b}^{2}}\right) \frac{\partial R_{s / b}}{\partial Z} \\
& +\left(R_{s / b}+\frac{1-R_{s / b}^{2}}{2 R_{s / b} \ln R_{s / b}}\right) \frac{1}{2 \sigma} \frac{\partial P}{\partial Z}, \\
& T_{R=R_{s / b}}^{z z}=-\left(R_{s / b}+\frac{1-R_{s / b}^{2}}{2 R_{s / b} \ln R_{s / b}}\right) \frac{1}{\sigma} \frac{\partial R_{s / b}}{\partial Z} \frac{\partial P}{\partial Z} .
\end{aligned}
$$

The dimensionless velocities, $U_{s}$ and $V_{s}$, at the surface of the rotating shaft, are given by

$$
\begin{aligned}
U_{s}= & \frac{d E}{d \tau} \cos (\Theta-\Phi)+E \frac{d \Phi}{d \tau} \sin (\Theta-\Phi) \\
& +2 R_{s / b}^{*} \sin \left(\Theta-\Theta^{*}-2 \tau\right), \\
V_{s}= & -\frac{d E}{d \tau} \sin (\Theta-\Phi)+E \frac{d \Phi}{d \tau} \cos (\Theta-\Phi) \\
& +2 R_{s / b}^{*} \cos \left(\Theta-\Theta^{*}-2 \tau\right),
\end{aligned}
$$

where

$$
\begin{aligned}
& R=\frac{r}{R_{b}}, \quad \Theta=\theta, \quad \Theta^{*}=\theta^{*}, \quad Z=\frac{z}{R_{b}}, \quad P=\frac{p}{P_{a}}, \\
& R_{s / b}=\frac{R_{s}}{R_{b}}, \quad R_{s / b}^{*}=\frac{R_{s}^{*}}{R_{b}}, \quad U_{s}=\frac{2 u_{s}}{R_{b} \omega}, \quad V_{s}=\frac{2 v_{s}}{R_{b} \omega}, \\
& E=\frac{e}{R_{b}}, \quad \sigma=\frac{\eta \omega}{P_{a}}, \quad \Phi=\phi, \quad \tau=\frac{\omega t}{2}, \quad T^{R R}=\frac{\tau^{r r}}{\omega \eta}, \\
& T^{\Theta \Theta}=\frac{\tau^{\theta \theta}}{\omega \eta}, \quad T^{R \Theta}=\frac{\tau^{r \theta}}{\omega \eta} .
\end{aligned}
$$

The dimensionless lubricant film thickness, $H$, is defined as $H \equiv 1-R_{s / b}$. Since $H$ is sufficiently small compared with unity, (11) reduce to

$$
\begin{gathered}
T_{R=R_{s / b}}^{R R} \approx-\frac{U_{s}}{H}, \\
T_{r=R_{s}}^{\Theta \Theta} \approx-\frac{H}{\sigma} \frac{\partial P}{\partial \Theta} \frac{\partial H}{\partial \Theta}-\frac{V_{s}}{2 H} \frac{\partial H}{\partial \Theta}+\frac{\partial V_{s}}{\partial \Theta}+U_{s}, \\
T_{R=R_{s / b}}^{R \Theta} \approx-\frac{H}{2 \sigma} \frac{\partial P}{\partial \Theta}-\frac{V_{s}}{2 H}+\frac{1}{2} \frac{\partial U_{s}}{\partial \Theta}-\frac{U_{s}}{2 H} \frac{\partial H}{\partial \Theta}, \\
T_{R=R_{s / b}}^{R Z} \approx \frac{1}{2} \frac{\partial U_{s}}{\partial Z}-\frac{H}{2 \sigma} \frac{\partial P}{\partial Z}-\frac{U_{s}}{2 H} \frac{\partial H}{\partial Z}+\frac{H}{2 \sigma} \frac{\partial P}{\partial Z}, \\
T_{R=R_{s / b}^{Z \Theta}}^{Z-\frac{1}{\sigma}} \frac{\partial P}{\partial \Theta} \frac{\partial H}{\partial Z}-\frac{V_{s}}{2 H} \frac{\partial H}{\partial Z}+\frac{1}{2} \frac{\partial V_{s}}{\partial Z}-\frac{H}{2 \sigma} \frac{\partial H}{\partial \Theta} \frac{\partial P}{\partial Z}, \\
T_{R=R_{s / b}}^{Z Z} \approx-\frac{H}{\sigma} \frac{\partial P}{\partial Z} \frac{\partial H}{\partial Z} .
\end{gathered}
$$

The dimensionless pressure, $P$, can be obtained through a modified Reynolds equation with curvature effect derived by Liu and Yoshihiro [6] as

$$
\begin{aligned}
\frac{\partial}{\partial \Theta}[ & \left.(1+H) H^{3} \frac{\partial P}{\partial \Theta}\right]+\frac{\partial}{\partial Z}\left[H^{3} \frac{\partial P}{\partial Z}\right] \\
= & -6 \sigma U_{s}\left(1-\frac{H}{2}\right) \\
& +3 \sigma V_{s}\left(1+H+\frac{5}{4} H^{2}\right) \frac{\partial H}{\partial \Theta} \\
& +3 \sigma\left(H+\frac{H^{2}}{2}\right) \frac{\partial V_{s}}{\partial \Theta} .
\end{aligned}
$$

At $Z=0$, the groove shape is symmetric, and at $Z=L$, it is assumed that the fluid is open to the atmosphere, so that boundary conditions of pressure are

$$
\begin{aligned}
& \frac{\partial P}{\partial Z}=0 \quad \text { at } Z=0, \\
& P=1.0 \quad \text { at } Z=L .
\end{aligned}
$$

In the numerical analysis, a spectral finite-difference scheme is used, and (15) is decomposed into each component of the Fourier series to the circumferential $\Theta$-direction. 


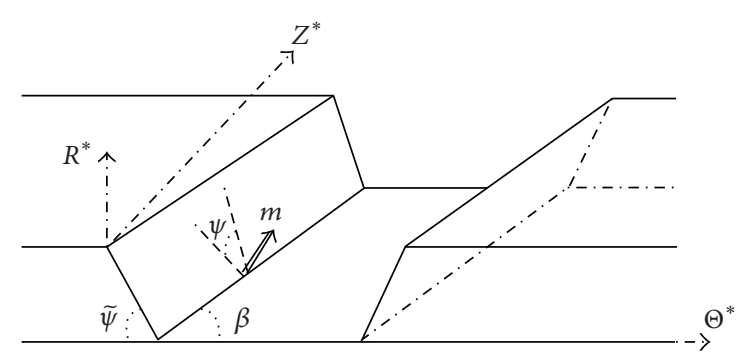

FIGURE 3: Trapezoidal surface of shaft.

\section{Load Capacity, Attitude Angle, and Friction Torque}

In a non inertial cylindrical coordinate system $S^{*}$, physical components of total stresses at $R^{*}=R_{s / b}^{*}$ are given by

$$
\begin{aligned}
& T_{R^{*}=R_{s / b}^{*}}^{R^{*} R^{*}}=\left(-\frac{P}{\sigma}+T_{R=R_{s / b}}^{R R}\right) \cos ^{2} \alpha-2 T_{R=R_{s / b}}^{R \Theta} \sin \alpha \cos \alpha \\
& +\left(-\frac{P}{\sigma}+T_{R=R_{s / b}}^{\Theta \Theta}\right) \sin ^{2} \alpha, \\
& T_{R^{*}=R_{s / b}^{*}}^{R^{*} \Theta^{*}}=\left(T_{R=R_{s / b}}^{R R}-T_{R=R_{s / b}}^{\Theta \Theta}\right) \cos \alpha \sin \alpha+T_{R=R_{s / b}}^{R \Theta} \cos 2 \alpha, \\
& T_{R^{*}=R_{s / b}^{*}}^{\Theta^{*} \Theta^{*}}=\left(-\frac{P}{\sigma}+T_{R=R_{s / b}}^{R R}\right) \sin ^{2} \alpha+2 T_{R=R_{s / b}}^{R \Theta} \sin \alpha \cos \alpha \\
& +\left(-\frac{P}{\sigma}+T_{R=R_{s / b}}^{\Theta \Theta}\right) \cos ^{2} \alpha, \\
& T_{R^{*}=R_{s / b}^{*}}^{R^{*} Z^{*}}=T_{R=R_{s / b}}^{R Z} \cos \alpha-T_{R=R_{s / b}}^{\Theta Z} \sin \alpha, \\
& T_{R^{*}=R_{s / b}^{*}}^{Z^{*} \Theta^{*}}=T_{R=R_{s / b}}^{R Z} \sin \alpha+T_{R=R_{s / b}}^{\Theta Z} \cos \alpha, \\
& T_{R^{*}=R_{s / b}^{*}}^{Z^{*} Z^{*}}=\frac{-P}{\sigma}+T_{R=R_{s / b}}^{Z Z},
\end{aligned}
$$

where $\alpha \approx \Theta-\Theta^{*}-2 \tau$, since $H \ll 1$.

For a trapezoidal surface of shaft in the coordinate system $S^{*}\left(r^{*}, \theta^{*}, z^{*}\right)$ as shown in Figure 3, a unit vector, $\mathbf{m}$, perpendicular to the trapezoidal surface is obtained as

$$
\mathbf{m}=(\cos \psi, \sin \psi,-\sin \psi \cos \beta),
$$

where the apparent trapezoidal angle of groove in $R^{*} \Theta^{*}$ cross-section, $\widetilde{\psi}$, is given as

$$
\cos \widetilde{\psi}=\frac{\cos \psi}{\sqrt{\sin ^{2} \beta+\cos ^{2} \psi \cos ^{2} \beta}} .
$$

Then, through some mathematic calculations, the $R^{*}$ - and $\Theta^{*}$-components of fluid force per unit area on the side of the trapezoidal surface are given

$$
\begin{aligned}
f_{R^{*}}^{d s}= & T_{R^{*}=R_{s / b}^{*}}^{R^{*} R^{*}} \cos \psi+T_{R^{*}=R_{s / b}^{*}}^{R^{*} \Theta^{*}} \sin \psi \sin \beta \\
& -T_{R^{*}=R_{s / b}^{*}}^{R^{*} Z^{*}} \sin \psi \cos \beta, \\
f_{\Theta^{*}}^{d s}= & T_{R^{*}=R_{s / b}^{*} R^{*}}^{\Theta^{*}} \cos \psi+T_{R^{*}=\Theta_{s / b}^{*}}^{\Theta^{*} \Theta^{*}} \sin \psi \sin \beta \\
& -T_{R^{*}=R_{s / b}^{*}}^{\Theta^{*} Z^{*}} \sin \psi \cos \beta,
\end{aligned}
$$

respectively, on the left surface in Figure 3. In addition, on the right surface, in (18)-(20), $\psi$ is replaced by $-\psi$. And the dimensionless horizontal component of load force, $F_{r}$, vertical component of load $F_{t}$, and friction torque of shaft, $T_{f}$, are

$$
\begin{aligned}
F_{r}= & \frac{f_{r}}{P_{a} R_{2}^{2}}=2 \sigma \int_{0}^{L} \int_{0}^{2 \pi}\left[f_{R^{*}}^{d s} \frac{\cos \left(\Theta^{*}+\Phi\right)}{\cos \psi} R_{s / b}^{*} d \Theta^{*}\right] d Z^{*} \\
& -2 \sigma \int_{0}^{L} \int_{0}^{2 \pi}\left[f_{\Theta^{*}}^{d s} \frac{\sin \left(\Theta^{*}+\Phi\right)}{\cos \psi} R_{s / b}^{*} d \Theta^{*}\right] d Z^{*}, \\
F_{t}= & \frac{f_{t}}{P_{a} R_{2}^{2}}=2 \sigma \int_{0}^{L} \int_{0}^{2 \pi}\left[f_{R^{*}}^{d s} \frac{\sin \left(\Theta^{*}+\Phi\right)}{\cos \psi} R_{s / b}^{*} d \Theta^{*}\right] d Z^{*} \\
& +2 \sigma \int_{0}^{L} \int_{0}^{2 \pi}\left[f_{\Theta^{*}}^{d s} \frac{\cos \left(\Theta^{*}+\Phi\right)}{\cos \psi} R_{s / b}^{*} d \Theta^{*}\right] d Z^{*}, \\
T_{f}= & \frac{t_{f}}{P_{a} R_{b}^{3}}=2 \sigma \int_{0}^{L} \int_{0}^{2 \pi}\left[\frac{f_{\Theta^{*}}^{d s}}{\cos \psi} R_{s / b}^{*} d \Theta^{*}\right] \times R_{s / b}^{*} d Z^{*} .
\end{aligned}
$$

Throughout (21), $\psi$ should be replaced as 0 if the location corresponds to groove or ridge.

The dimensionless load capacity, $W$, is given by

$$
W=\sqrt{F_{r}^{2}+F_{t}^{2}}
$$

and the attitude angle, $\varphi$, is

$$
\varphi=\tan ^{-1}\left(\frac{F_{t}}{F_{r}}\right)
$$

\section{Results and Discussion}

Figure 4 shows the relation between load capacity and eccentricity in case of $n_{g}=20, \beta=21.8$ degrees, $a_{r} / a_{g}=1.0, L=$ $R_{b}, \Omega=0, \Lambda \equiv 6 \sigma \times\left(R_{b} / C_{r}\right)^{2}=0.029$. The numerical results are in good agreement with the experimental data by Hirs [7]. The trapezoidal groove shape was not in the focus of Hirs. In the trapezoidal angle is fixed at 45 degrees, because the load capacity of Hirs model changes slight with trapezoidal angle from 10 to 80 degrees as shown in Figure 5. 


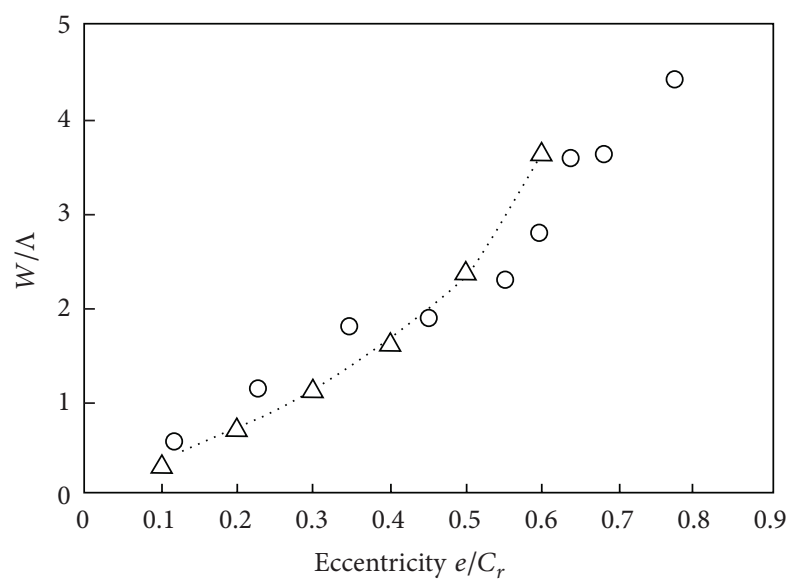

O Experimental data by Hirs

$\triangle$ Numerical results

FIgURE 4: Comparison of load capacity with experimental data.

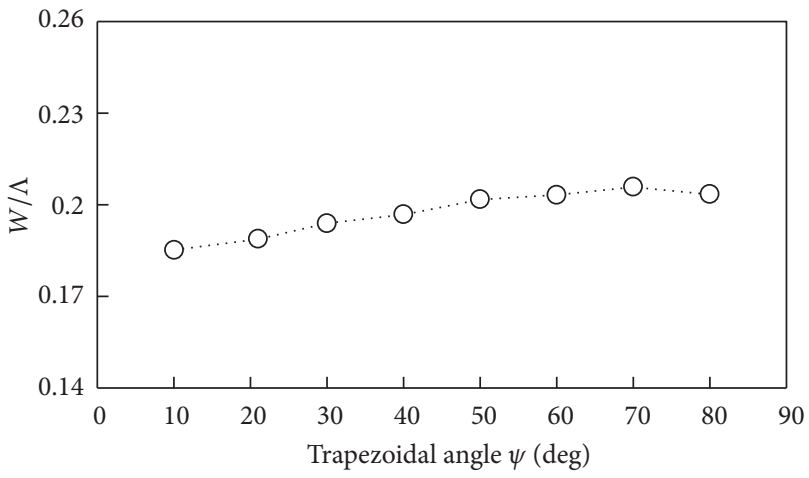

FIGURE 5: Variations of load capacity with trapezoidal angle in case of $e / C_{r}=0.1$.

The effects of trapezoidal groove are estimated as

$$
\varepsilon_{w}=\frac{W-W^{g}}{W^{g}}, \quad \varepsilon_{\varphi}=\frac{\varphi-\varphi^{g}}{\varphi^{g}}, \quad \varepsilon_{T}=\frac{T_{f}-T_{f}^{g}}{T_{f}^{g}},
$$

where quantities with a superscript " $g$ " correspond to those without trapezoidal surface, only pressure being taken into account, that is,

$$
\begin{gathered}
F_{r}^{g}=2 \sigma \int_{0}^{L} \int_{0}^{2 \pi} P \cos \left(\Theta^{*}-\Phi\right) d \Theta^{*} d Z^{*}, \\
F_{t}^{g}=2 \sigma \int_{0}^{L} \int_{0}^{2 \pi} P \sin \left(\Theta^{*}-\Phi\right) d \Theta^{*} d Z^{*}, \\
W^{g}=\sqrt{\left(F_{r}^{g}\right)^{2}+\left(F_{t}^{g}\right)^{2}}, \\
\varphi^{g}=\tan ^{-1}\left(\frac{F_{t}^{g}}{F_{r}^{g}}\right), \\
T_{f}^{g}=2 \sigma \int_{0}^{L} \int_{0}^{2 \pi} T_{R^{*}=R_{s / b}^{*}}^{R^{*}} \times R_{s / b}^{* 2} d \Theta^{*} d Z^{*} .
\end{gathered}
$$

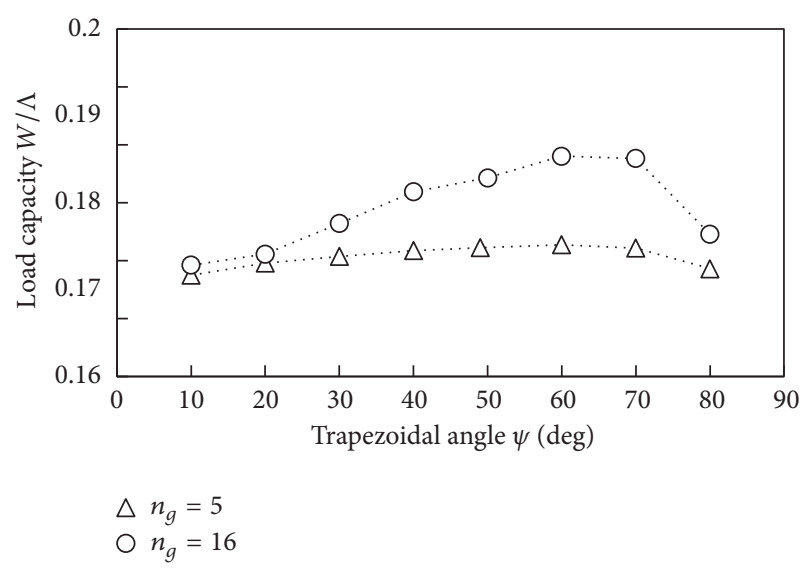

FIGURE 6: Variations of load capacity with trapezoidal angle.

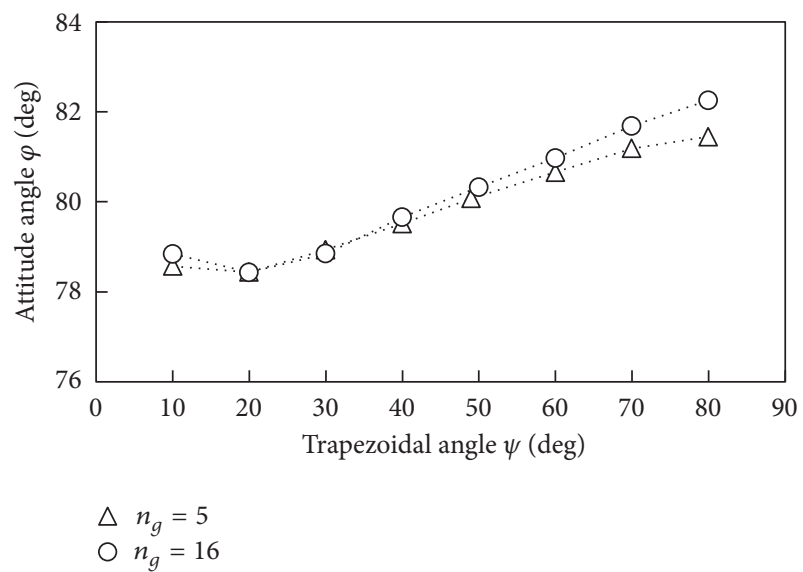

FIGURE 7: Variations of attitude angle with trapezoidal angle.

In case of $\delta=C_{r}, C_{r} / R_{b}=0.005, \beta=$ 30 degrees, $a_{r} / a_{g}=1.0, \Lambda=1.0, L=R_{b}, \Omega=0, e / C_{r}=0.3$, the load capacity, attitude angle, and friction torque against 2 cases of groove number are evaluated as a function of trapezoidal angles as shown in Figures 6, 7, and $8\left(n_{g}=\right.$ 5 and $\left.n_{g}=16\right)$. The characteristics of bearing for $n_{g}=$ 16 are more sensitive than those for $n_{g}=5$. As long as trapezoidal angle is smaller than 60 degrees, the load capacity becomes large with trapezoidal angle, but in the case that trapezoidal angle is larger than 60 degrees, the load capacity decreases with the trapezoidal angle. The attitude angle is slightly decreased with the trapezoidal angle if the trapezoidal angle is smaller than 20 degrees, and if the trapezoidal angle is larger than 20 degrees, the attitude angle increases with the trapezoidal angle. The friction torque increases with the trapezoidal angle.

Figures 9, 10, and 11 show effects of the trapezoidal groove shape on the journal bearing characteristics. The effects of trapezoidal shape on the load capacity and attitude angles are vanishingly small (less than 1\%), where the trapezoidal shape has large effect on the friction torque. 


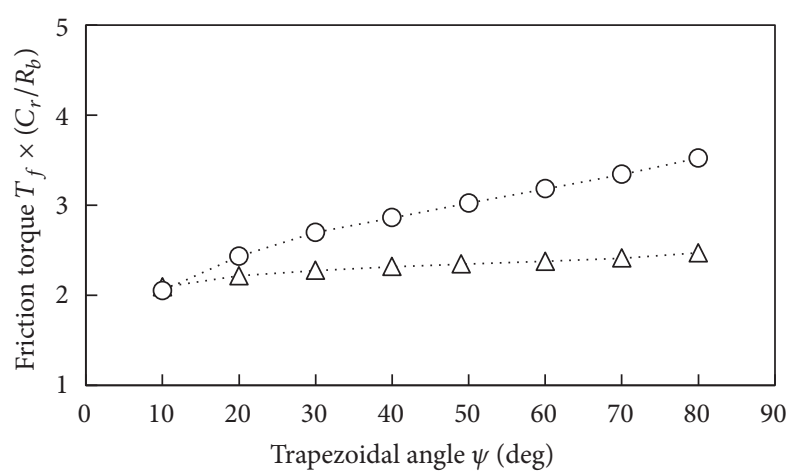

$\triangle n_{g}=5$

$\bigcirc n_{g}=16$

Figure 8: Variations of friction torque with trapezoidal angle.

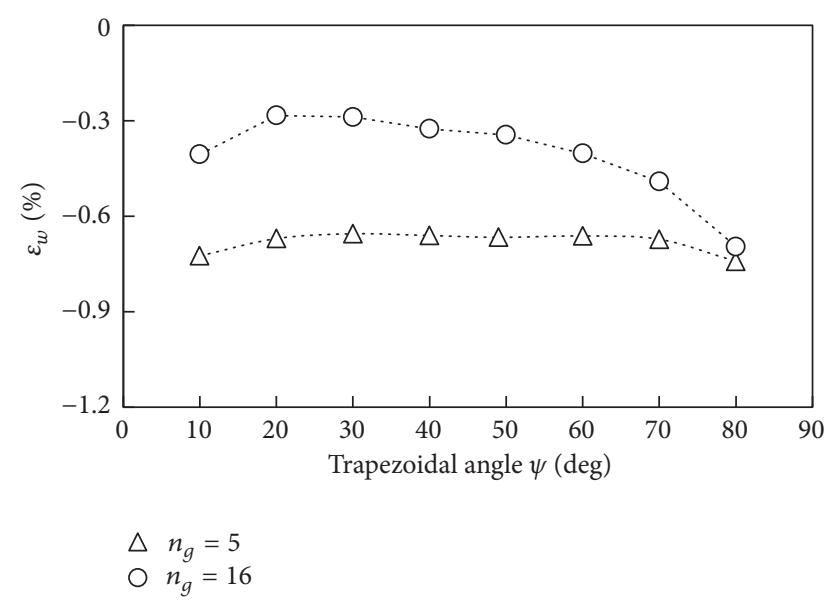

Figure 9: Effects of trapezoidal shape on the load capacity with trapezoidal angle.

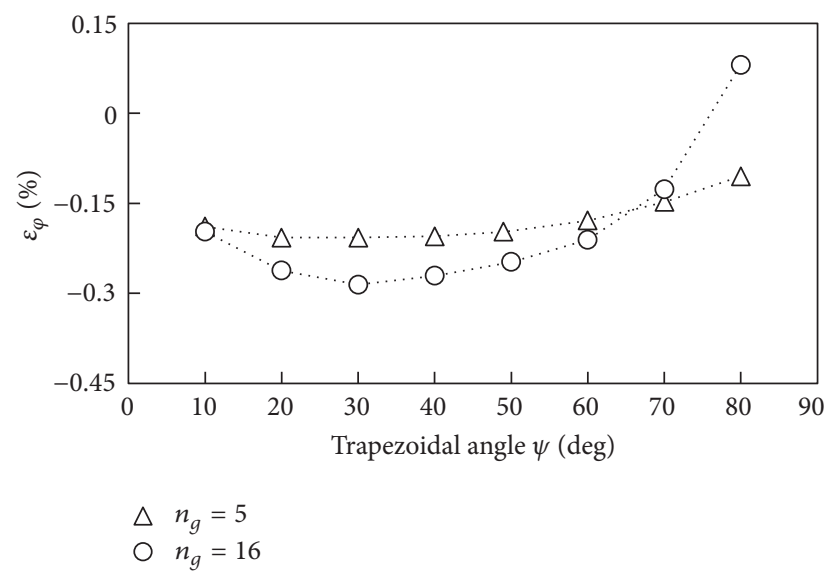

Figure 10: Effects of trapezoidal shape on the attitude angle with trapezoidal angle.

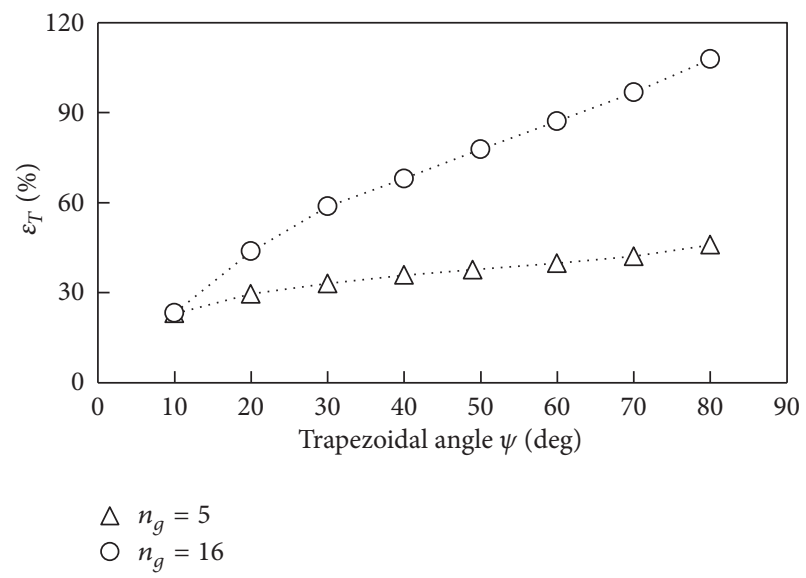

FIGURE 11: Effects of trapezoidal shape on the friction torque with trapezoidal angle.

\section{Conclusions}

(1) The characteristics of a self-acting fluid-lubricated herringbone grooves journal bearing with trapezoidal groove are calculated.

(2) The influences of trapezoidal groove shape on the journal-bearing characteristics such as the variation of load capacity, attitude angle, and friction torque for various grooves geometry are discussed. It shows that the effects on the load capacity and attitude angles are vanishingly small, but the effects have unignorable function on the friction torque, and the effects of trapezoidal groove become larger with trapezoidal angle increase.

\section{Nomenclature}

$a_{g}, a_{r}: \quad$ Grooves width, ridge width

$C_{r}$ : $\quad$ Bearing clearance

$e, E: \quad$ Eccentricity, dimensionless eccentricity

$f_{R^{*}}^{d s}, f_{\Theta^{*}}^{d s}$ : Dimensionless the $R^{*}$-, and $\Theta^{*}$-components of fluid force per unit area which acts on surface of shaft

$F_{r}, F_{t}$ : Dimensionless horizontal component, vertical component of load

$H: \quad$ Dimensionless fluid film thickness

$l, L: \quad$ Bearing length, dimensionless bearing length

$n_{g}: \quad$ Number of grooves

m: A unit vector which is perpendicular to the trapezoidal surface

$p, P: \quad$ Pressure, dimensionless pressure

$P_{a}: \quad$ Atmospheric pressure

$r, \theta, z: \quad$ Inertial coordinates

$R_{b}: \quad$ Radius of bearing

$R_{s 0}$ : $\quad$ Radius of shaft without grooves

$R_{s}$ : $\quad$ Radial component of coordinate at surface of shaft 
$R_{s / b}$ : Dimensionless radial component of coordinate at surface of shaft

$t, \tau: \quad$ Time, dimensionless time

$T_{R=R_{s / b}}^{R R}: \quad$ Dimensionless $R R$-component of viscous stress on the surface of shaft (at $R=R_{s / b}$ )

$T_{R=R_{s / b}}^{R \Theta}: \quad$ Dimensionless $R \Theta$-component of viscous stress on the surface of shaft (at $R=R_{s / b}$ )

$T_{R=R_{s / b}}^{\Theta \Theta}: \quad$ Dimensionless $\Theta \Theta$-component of viscous stress on the surface of shaft (at $R=R_{s / b}$ )

$T_{R=R_{s / b}}^{Z \Theta}: \quad$ Dimensionless $\Theta R$-component of viscous stress on the surface of shaft (at $R=R_{s / b}$ )

$T_{R=R_{s / b}}^{R Z}$ : $\quad$ Dimensionless $R Z$-component of viscous stress on the surface of shaft (at $R=R_{s / b}$ )

$T_{R=R_{s / b}}^{Z Z}: \quad$ Dimensionless $Z Z$-component of viscous stress on the surface of shaft (at $R=R_{s / b}$ )

$T_{f}$ : Dimensionless friction torque of rotating shaft

$u_{s}: \quad$ Circumferential velocity at surface of rotating shaft

$U_{s}: \quad$ Dimensionless circumferential velocity at surface of rotating shaft

$v_{s}: \quad$ Radial velocity at surface of rotating shaft

$V_{s}$ : Dimensionless radial velocity at surface of rotating shaft

$v_{r}, v_{\theta}, v_{z}: \quad$ Velocity components of lubricant fluid

$W: \quad$ Dimensionless load capacity of bearing

$\beta$ : $\quad$ Groove angle

$\delta, \Delta: \quad$ Groove depth, dimensionless groove depth

$\eta$ : $\quad$ Viscosity of fluid

$\Lambda$ : $\quad$ Bearing number

$\sigma: \quad$ Dimensionless number

$\tau^{i j}, T^{i j}: \quad$ Viscous stress, dimensionless viscous stress

$\varphi: \quad$ Attitude angle of shaft

$\psi: \quad$ Trapezoidal angle of groove

$\widetilde{\psi}: \quad$ Apparent trapezoidal angle of groove in

$R^{*} \Theta^{*}$ cross-section

$\phi, \Phi: \quad \quad$ Angle between the fixed axis of abscissa

$(\theta=0)$ and the axis of eccentricity,

dimensionless angle

$\omega: \quad$ Rotation velocity of shaft

$\Omega: \quad$ Rwirl velocity of shaft

superscript ${ }^{*}$ : Noninertial coordinate.

\section{References}

[1] J. H. Vohr and C. H. T. Pan, "On The Spiral-grooved, Self-acting Gas Bearing," MTI63TR52, 1963.

[2] B. J. Hamrock and D. P. Fleming, "Optimization of Self-acting Herringbone Grooved Journal Bearings for Maximum Radial Load Capacity," NASA-TM-X-52945, 1971.

[3] S. Murata, Y. Miyake, and N. Kawabata, "Two-dimensional analysis of herringbone groove journal bearing," Bulletin of the JSME, vol. 23, no. 181, pp. 1980-1987, 1980.

[4] D. Bonneau and J. Absi, "Analysis of aerodynamic journal bearings with small number of herringbone grooves by finite element method," Journal of Tribology, vol. 116, no. 4, pp. 698-704, 1994.
[5] G. H. Jang and D. I. Chang, "Analysis of a hydrodynamic herringbone grooved journal bearing considering cavitation," Journal of Tribology, vol. 122, no. 1, pp. 103-109, 2000.

[6] J. Liu and Y. Mochimaru, "Analysis of oil-lubricated herringbone grooved journal bearing with trapezoidal cross-section, using a spectral finite difference method," Journal of Hydrodynamics $B$, vol. 22, no. 5, supplement 1, pp. 408-412, 2010.

[7] G. G. Hirs, "The load capacity and stability characteristics of hydro-dynamic grooved journal bearing," ASLE Transaction, vol. 8, pp. 296-305, 1965. 

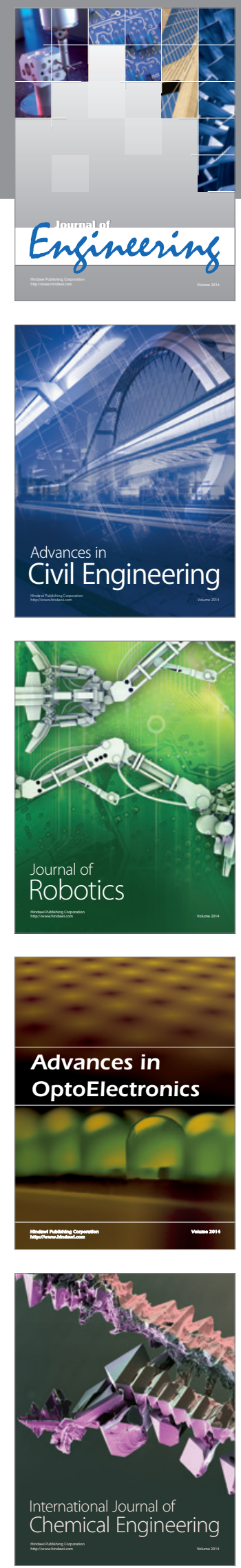

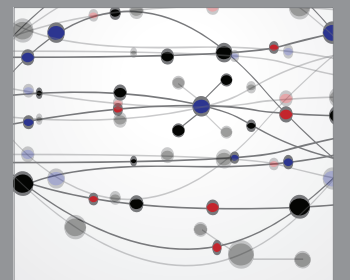

The Scientific World Journal
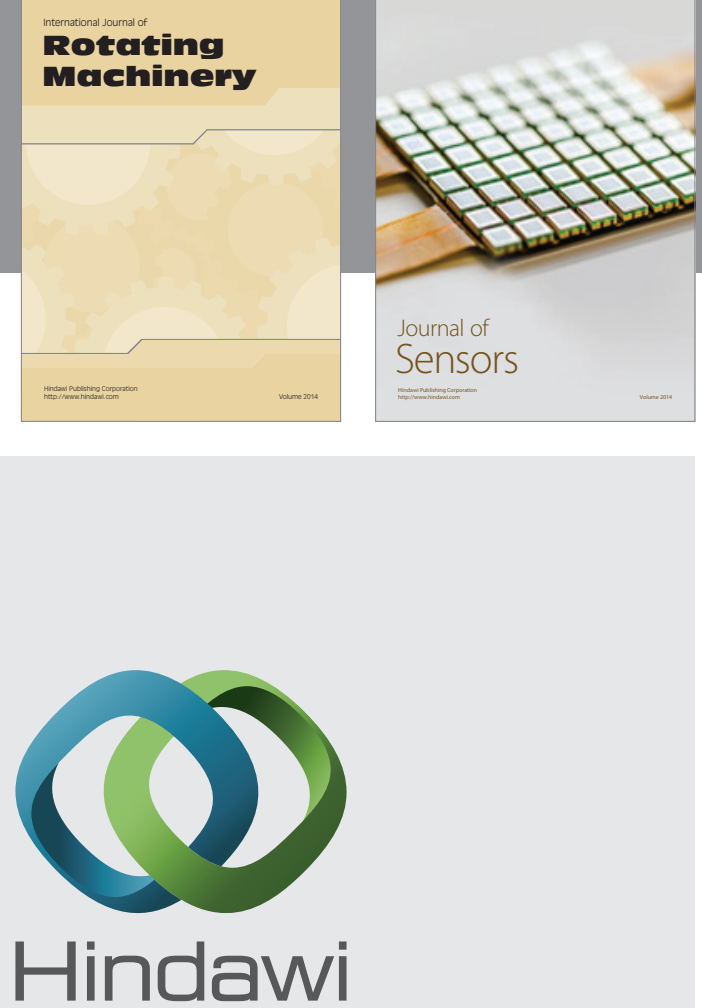

Submit your manuscripts at http://www.hindawi.com
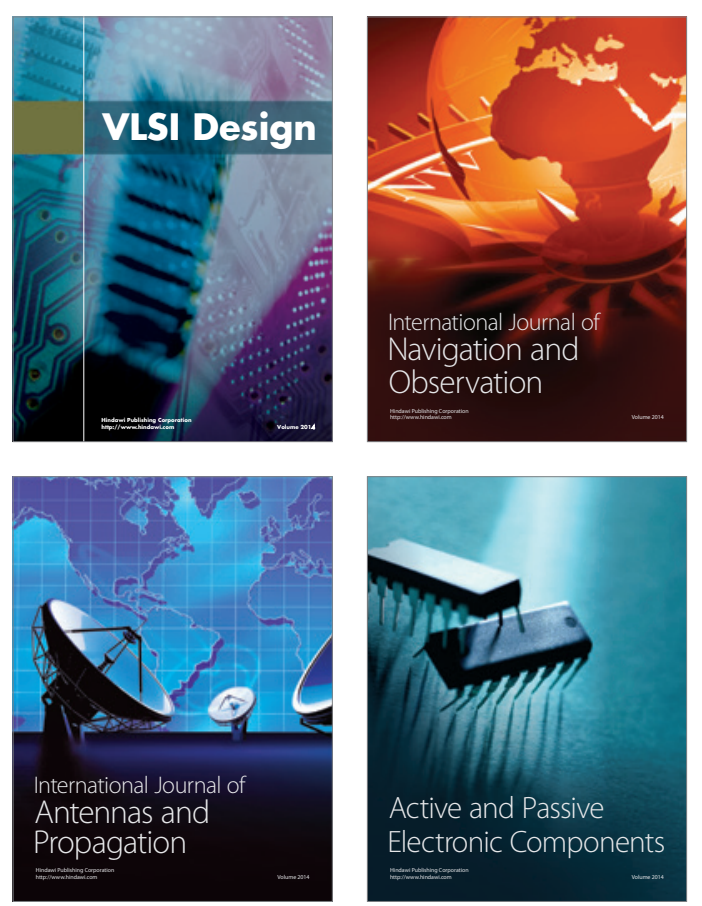
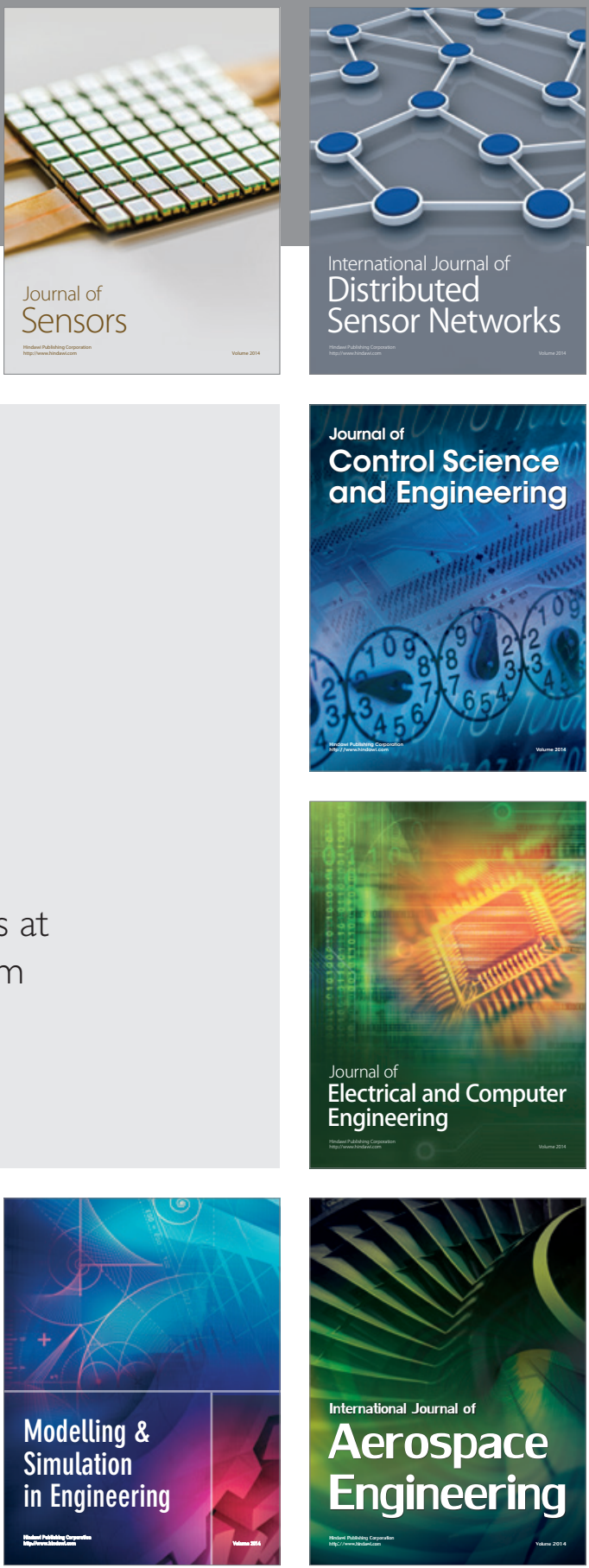

Journal of

Control Science

and Engineering
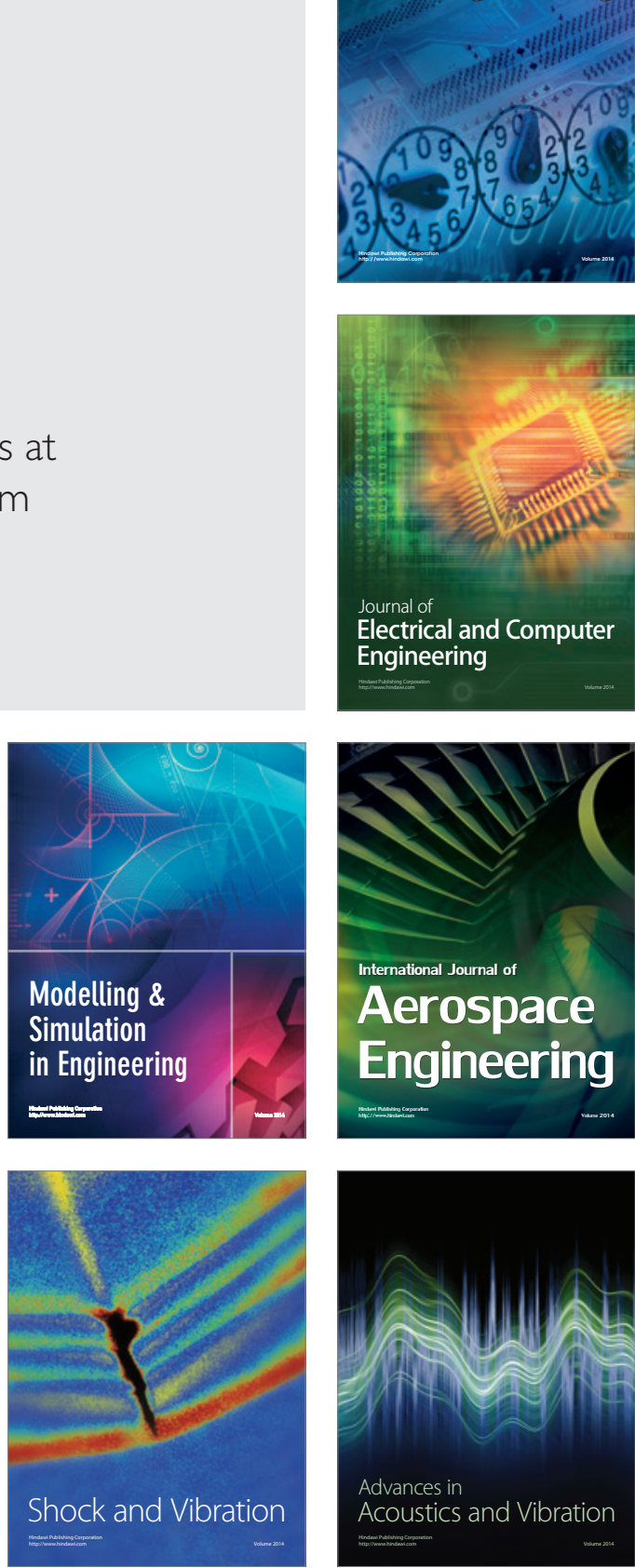